\title{
Aproximación conceptual a la separación del dióxido de corbono en corrientes de combustión
}

\author{
Carbon Dioxide Separation from Combustion Stream: A \\ Conceptual Approach
}

\section{Resumen}

El dióxido de carbono $\left(\mathrm{CO}_{2}\right)$ es considerado uno de los principales gases causantes del efecto invernadero, responsable de los fenómenos de calentamiento global y cambio climático; por ello se han propuesto distintas estrategias de captura y almacenamiento físico o químico de $\mathrm{CO}_{2}$, especialmente en las emisiones provenientes de corrientes de combustión. Este documento presenta, en primer lugar, una revisión de los procesos de captura de $\mathrm{CO}_{2}$ en procesos de combustión, y, luego, dos escenarios de estudio para la captura de $\mathrm{CO}_{2}$ en un proceso de poscombustión usando metanol como combustible. En el primer escenario se separa el

\begin{abstract}
The carbon dioxide $\left(\mathrm{CO}_{2}\right)$ is considered to be one of the major green house gases effect's causes, responsible for most of both global warming and climate change. Due to the $\mathrm{CO}_{2}$ emissionmeaning, several physic and chemical capture and storage processes have been proposed, especially from combustion procedures. In this work, carbon capture technologies were reviewed. Here, two scenarios for post-combustion carbon capture from methanol combustion are presented. The first one is a chemical absorption separation with monoethanolamine. The second one is a separation by the distillation tower. The results indicate that both technologies are appropriated,
\end{abstract}

\footnotetext{
* M.Sc. Universidad Nacional de Colombia, Sede Manizales (Colombia).mjvalenciab@unal.edu.co

** Ph.D. Universidad Nacional de Colombia, Sede Manizales (Colombia). ccardonaal@unal.edu.co
} 
$\mathrm{CO}_{2}$ utilizando absorción con monoetanolamina, y en el segundo se utiliza destilación. Los resultados indican que ambas tecnologías son apropiadas, pero que el rendimiento es mayor con la destilación.

Palabras clave: Dióxido de carbono, Calentamiento global, Efecto invernadero, Captura de $\mathrm{Co}_{2}$, Absorción química, destilación. but the distillation has higher yields than the chemical absorption.

Keywords: Carbon Dioxide, Global Warming, Green House Effect, $\mathrm{Co}_{2}$ Capture, Combustion, Separation Processes, Chemical Absorption, Distillation. 


\section{INTRODUCCIÓN}

Las emisiones de dióxido de carbono $\left(\mathrm{CO}_{2}\right)$ se consideran la principal causa del aumento de la temperatura de la Tierra en los últimos tiempos, debido a la capacidad de este compuesto de afectar el balance radiante del planeta. Aunque existen en la atmósfera otros gases con mayor capacidad de retener la radiación (por ejemplo, el metano, el óxido nitroso y los halocarbonados), la cantidad de emisiones de $\mathrm{CO}_{2}$ lo convierten en la principal causa del calentamiento global y el cambio climático [1]. Estas emisiones están relacionadas con esquemas de producción y uso de energía, en mayor medida, y con los procesos químicos e industriales y las operaciones unitarias, en menor medida [2]. El uso de combustibles fósiles como fuente de energía genera las mayores cantidades de $\mathrm{CO}_{2}$, debido al contenido de carbono, las cuales se depositan directamente en la atmósfera.

Dado que las emisiones de $\mathrm{CO}_{2}$ se originan en procesos energéticos que sustentan el desarrollo y crecimiento económico [3], la comunidad científica ha propuesto otras alternativas para mitigar sus consecuencias. La captura de $\mathrm{CO}_{2}$ para inyectarlo en grandes cantidades a altas presiones en pozos petroleros vacíos o en el océano, conocida como captura geológica, ha sido la primera línea de posibles alternativas de mitigación [4]. Otras propuestas consisten en el uso de $\mathrm{CO}_{2}$ como materia prima para obtener productos de valor agregado [5]. En esta línea de propuestas se ha indicado que entre los posibles usos de este componente están su aplicación en extracción supercrítica, como refrigerante y en uso agrícola, entre otros $[6,7]$.

La captura de $\mathrm{CO}_{2}$, independientemente de su posterior almacenamiento o uso como materia prima, requiere del análisis tecnológico de sistemas de separación del $\mathrm{CO}_{2}$ de distintas corrientes. Considerando que las mayores concentraciones de $\mathrm{CO}_{2}$ son subproductos del sector energético, por las corrientes de combustión, en el presente documento se realiza una revisión y simulación de separación del dióxido de carbono proveniente de corrientes de combustión.

\section{Tecnologías de Combustión Con Captura de $\mathrm{CO}_{2}$}

La combustión es un tipo de reacción química mediante la cual un material reacciona con oxígeno para obtener cantidades apreciables de energía, ya sea directamente o a través de un vector energético [8,9]. Debido a las temperaturas a las cuales se lleva a cabo la reacción, los componentes finales están, en su mayoría, en estado gaseoso. La composición de los gases de combustión depende de la materia prima o de la definición del proceso en el cual se lleve a cabo la combustión [10, 11]. Los materiales más complejos permiten obtener, como gases de combustión, mezclas de óxidos de carbono, nitrógeno y azufre y compuestos orgánicos volátiles diferentes del metano, en distintos estados de oxidación, dependiendo del oxígeno alimentado y de la temperatura de reacción. Adicionalmente, moléculas complejas generan productos intermedios que dificultan el análisis, por ejemplo, dioxinas, hidrocarburos, ésteres y aldehídos $[12,13]$.

La composición de las corrientes de combustión en procesos convencionales implica bajas concentraciones en volumen de $\mathrm{CO}_{2}$, y no existe ningún tipo de separación o captura diferente de la regulación de los niveles de los óxidos de nitrógenoyazufre.Enlosesquemasdecombustión con captura de $\mathrm{CO}_{2}$ se plantean tres posibilidades de proceso: Captura precombustión, captura poscombustión y oxycombustión [14]. Las principales características de las posibilidades de combustión-captura son: 
- Captura precombustión: Consiste en convertir cualquier combustible en gas de síntesis. $\mathrm{El} \mathrm{CO}_{2}$ se separa del gas de síntesis, y la combustión se lleva a cabo con hidrógeno [15]. Los esquemas de precombustión están aumentando en los últimos tiempos, debido a la eficiencia en la recuperación energética cuando el combustible se convierte en gas de síntesis previo a la liberación de su contenido energético. Este esquema es particularmente útil para la cogeneración energética a partir de residuos agroindustriales.

- Captura poscombustión: En este caso la combustión se lleva a cabo y el $\mathrm{CO}_{2}$ se recupera de los gases producto de la combustión [16, 17]. Este esquema de captura se acoplaría con mayor facilidad en las industrias, ya que la mayoría de tecnologías convencionales para la producción energética se llevan a cabo por combustión directa del combustible.

- Oxycombustión: Este proceso está basado en una combustión con oxígeno puro para obtener una corriente de $\mathrm{CO}_{2}$ enriquecida, con mayor probabilidad de una separación de alta eficiencia [4]. La oxycombustión corresponde al esquema tecnológico más avanzado en combustión con captura de $\mathrm{CO}_{2}$. La dificultad de su implementación radica en la separación del oxígeno de las corrientes de aire.

Cada uno de los procesos de combustión con captura de $\mathrm{CO}_{2}$ puede ser combinado con una ruta de separación propia. En la tabla 1 se resumen las tecnologías de separación asociadas con los procesos $[3,18]$.

TABLA 1

RUTAS CONOCIDAS PARA LA SEPARACIÓN DE ACUERDO CON LA COMBUSTIÓN

\begin{tabular}{|l|l|l|l|}
\hline $\begin{array}{c}\text { Tipo de sistema } \\
\text { combustión-cap- } \\
\text { tura }\end{array}$ & $\begin{array}{l}\text { Principales compuestos } \\
\text { de la corriente separar }\end{array}$ & $\begin{array}{l}\text { Tecnología convencional } \\
\text { de separación de dióxido } \\
\text { de carbono }\end{array}$ & $\begin{array}{c}\text { Nuevas tecnologías de } \\
\text { separación de dióxido de } \\
\text { carbono }\end{array}$ \\
\hline Precombustión & $\begin{array}{l}\text { Dióxido de carbono, hi- } \\
\text { drógeno }\end{array}$ & Absorción química & $\begin{array}{l}\text { Membranas. Absorción fisicoquí- } \\
\text { mica }\end{array}$ \\
\hline Poscombustión & $\begin{array}{l}\text { Dióxido de carbono, } \\
\text { óxidos de nitrógeno y } \\
\text { óxidos de azufre }\end{array}$ & Absorción & $\begin{array}{l}\text { Captura de carbono por medio de } \\
\text { ciclos carbonatados. Membranas }\end{array}$ \\
\hline Oxycombustión & $\begin{array}{l}\text { Oxígeno, dióxido de } \\
\text { carbono, óxidos de } \\
\text { nitrógeno, óxidos de } \\
\text { azufre, agua }\end{array}$ & Separación criogénica & Membranas. Absorción \\
\hline
\end{tabular}

Cada una de las tecnologías de combustióncaptura presenta ventajas y desventajas; por ejemplo, la captura precombustión se basa en tecnologías de separación conocidas y probadas, lo que disminuye costos en términos de compresión, menor contenido de óxidos de azufre y nitrógeno y una amplitud de aplicación en cuanto al combustible. No obstante, la conversión a gas de síntesis afecta los rendimientos globales del proceso, y se requiere una línea de tratamiento especial para separar o utilizar las corrientes de nitrógeno asociada al proceso.

En el caso de la captura poscombustión, las tecnologías de aplicación son comerciales, y los requerimientos de modificaciones son menores con respecto a los otros esquemas; 
en contraste, se presenta mayor cantidad de impurezas en el proceso, se requieren entradas adicionales de materia y los costes energéticos por compresión son mayores. En la actualidad se tienen tres procesos comerciales para captura poscombustión, usando aminas: el proceso Kerr-McGee/ABB Lummus Crest, el proceso Fluor Daniel ECONAMINE y el proceso Kansai Electric Power o Mitsubishi Heavy Industries Ltd KEPCO/MHT [14]; sin embargo, otros esfuerzos investigativos se requieren para encontrar otras alternativas comerciales a las aminas, como medio de absorción del $\mathrm{CO}_{2}$.

Por su parte, la oxycombustión es el esquema de combustión-captura más reciente. Las grandes temperaturas que se alcanzan en la combustión constituyen su principal desventaja, ya que para controlarlas se requiere recircular los gases, aumentando los tamaños de reactor y reduciendo las eficiencias. Debido a su condición novedosa, es una tecnología que se desarrolla, y los grandes proyectos se encuentran a nivel piloto [19].

\section{Caso de estudio: Captura POSCOMBUSTIÓN DEL METANO}

El metanol es considerado uno de los posibles alcoholes para mezclas combustibles con gasolina, debido a su capacidad de aumentar el octanaje de las mezclas [20]; adicionalmente, como puede ser sintetizado de diversidad de materias primas, incluyendo el $\mathrm{CO}_{2}$, se ha considerado una posible materia prima energética con ciclo cerrado [21]. El uso de metanol como materia prima de un proceso de combustióncaptura podría considerarse una propuesta innovadora, ya que permite integrar el uso de un combustible alternativo con un proceso de captura apropiado en un contexto de reducción de emisiones. Los esquemas de mitigación en Colombia incluyen el uso de biocombustibles, que en la actualidad se reducen a bioetanol y biodiésel. Debido a la naturaleza de la cadena de suministro, existen algunas discusiones enfocadas a la seguridad alimentaria y al ahorro de emisiones que realmente supone el uso de mezclas de biocombustibles. No obstante, las tecnologías de captura en la combustión no han sido estudiadas en conjunto con las políticas de reducción. La captura de carbono podría ser una herramienta apropiada para cumplir con las metas gubernamentales. A continuación se describe este proceso y el estudio de dos tecnologías de separación del $\mathrm{CO}_{2}$.

\section{A. Descripción de los procesos}

La separación del $\mathrm{CO}_{2}$ de corrientes de combustión fue simulada empleando Aspen Plus v7.1 como herramienta principal. Se probaron dos tecnologías para la separación del $\mathrm{CO}_{2}$ previo a un proceso de combustión convencional: absorción química como monoetanolamina y destilación.

En el caso de la separación por absorción química (Fig. 1), se comienza desde la combustión completa del metanol. La corriente de gases se acondiciona para entrar al absorbedor, donde la monoetanolamina [17] reacciona con el dióxido de carbono de forma electrolítica, formando carbamato de etanol; esto con el fin de separarlo de la corriente y obtener un vapor enriquecido en dióxido de carbono. Posteriormente se entra a una torre de desorción y se acondiciona por medio de compresión y enfriamiento a las condiciones de disposición final. 


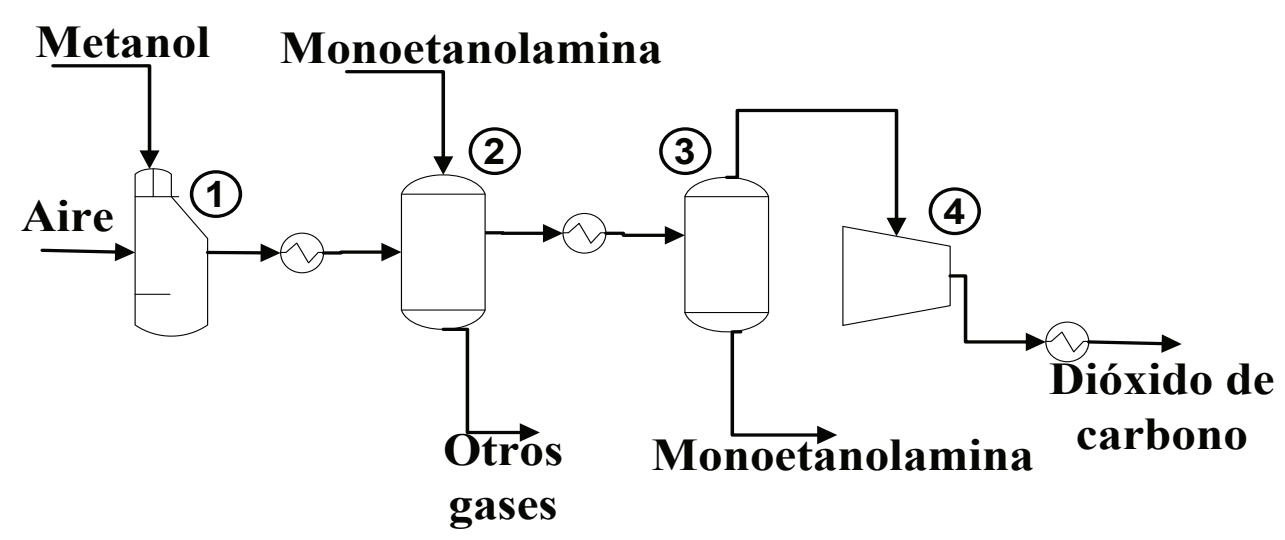

Fig. 1. Proceso de combustión y separación del $\mathrm{CO}_{2}$ por absorción química 1. Reactor de combustión, 2. Absorbedor, 3. Torre de desorción, 4. Compresor.

Para la ruta de separación por destilación, la quema del metanol es simulada, y los gases de salida son enfriados para entrar a una torre de destilación. La corriente rica en dióxido de carbono pasa a un compresor y a un enfriador con el fin de obtener las condiciones normales de manipulación y quedar adecuado para su disposición final.

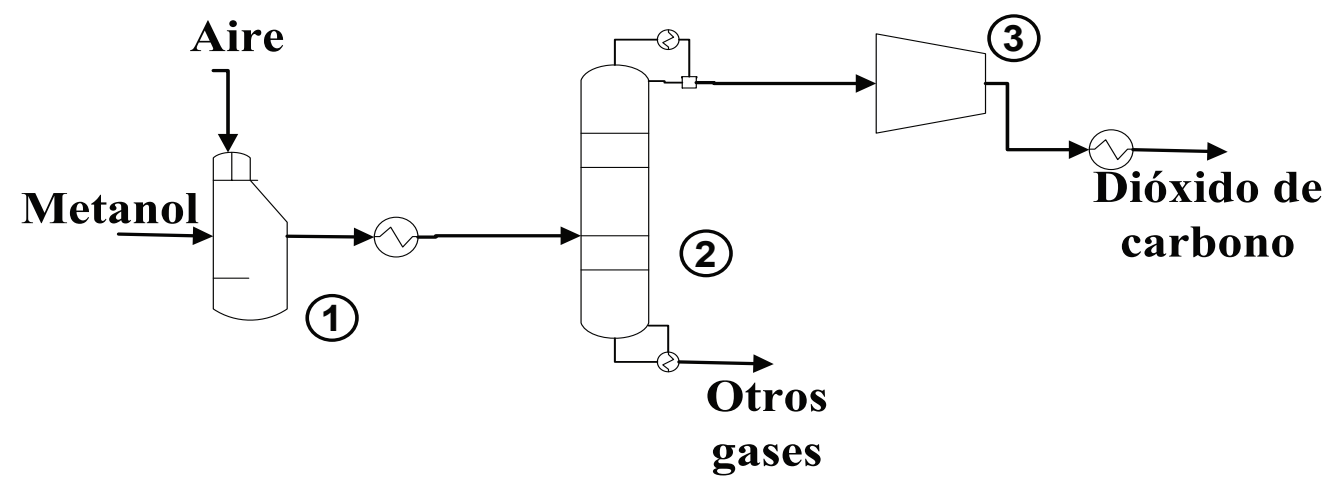

Fig. 2. Proceso de combustión y separación del $\mathrm{CO}_{2}$ por destilación

1. Reactor de combustión, 2. Absorbedor, 3. Torre de desorción, 4. Compresor.

Las principales condiciones de operación empleadas en la simulación se presentan en la tabla 2.

TABLA 2

CONDICIONES DE SIMULACIÓN

\begin{tabular}{|l|l|l|}
\hline \multicolumn{1}{|c|}{ Parámetro } & \multicolumn{1}{c|}{ Destilación } & \multicolumn{1}{c|}{ Absorción } \\
\hline Metanol entrada $(\mathrm{kmol} / \mathrm{h})$ & 100 & 100 \\
\hline Oxígeno entrada $(\mathrm{kmol} / \mathrm{h})$ & 150 & 150 \\
\hline Monoetanolamina $(\% \mathrm{~mol})$ & & 30 \\
\hline Corriente de absorbente $(\mathrm{kmol} / \mathrm{h})$ & & 100 \\
\hline Temperatura reactor $\left({ }^{\circ} \mathrm{C}\right)$ & 300 & 300 \\
\hline Presión del proceso $(\mathrm{bar})$ & 1,01325 & 1,01325 \\
\hline Presión final $($ bar $)$ & 200 & 200 \\
\hline Eficiencia isentrópica del compresor & 0,7 & 0,7 \\
\hline Eficiencia mecánica del compresor & 0,8 & 0,8 \\
\hline Modelo termodinámico & NRTL & ELECNRTL \\
\hline Temperatura final del $\mathrm{CO}_{2}\left({ }^{\circ} \mathrm{C}\right)$ & 24,68 & 29,17 \\
\hline
\end{tabular}




\section{B. Resultados y discusión}

Las condiciones a las cuales se llevaron a cabo las simulaciones son idénticas hasta el cambio en el método de separación. Debido a la relación de alimentación elegida para la combustión
(1:1,5 metanol:oxígeno en este caso) esta fue completa, lo que implica que la concentración de $\mathrm{CO}_{2}$ es alta y la ruta de separación puede ser relativamente sencilla. Los resultados obtenidos para ambas rutas se muestran en la tabla 3.

TABLA 3

RESULTADOS DE LA SIMULACIÓN

\begin{tabular}{|l|c|c|}
\hline \multicolumn{1}{|c|}{ Parámetro } & Destilación & Absorción \\
\hline Recuperación de $\mathrm{CO}_{2}$ & $90 \%$ & $70 \%$ \\
\hline Etapas de la columna de destilación & 2 & \\
\hline Etapas de la columna de absorción & & 10 \\
\hline Etapas de la columna de desorción & & 10 \\
\hline
\end{tabular}

En un caso como el simulado, las condiciones de separación con mejor relación costobeneficio están directamente relacionadas con aquel método que requiera menor cantidad de dispositivos adicional al porcentaje de recuperación. Con la destilación se obtuvo un mayor porcentaje de recuperación que con la absorción química. Pero una torre de destilación es ineficiente desde el punto de vista energético, a diferencia del absorbedor, donde las condiciones de separación están gobernadas por la termodinámica de las corrientes de entrada y de salida y por la disponibilidad de los reactivos. En este caso de combustión completa la destilación permite obtener resultados positivos, pero en el caso de combustiones incompletas, tanto la absorción química como la destilación pueden presentar rendimientos bajos por la presencia de compuestos con estados intermedios de oxidación.

\section{ObSERVaciones Finales}

La clave del proceso de separación del $\mathrm{CO}_{2}$ está muy relacionada con la eficiencia en el proceso de combustión y de la materia prima. Si se garantizan condiciones de combustión donde la cantidad de componentes indeseados sea mínima o inexistente (como en este caso), la separación se convierte en un problema de solución convencional; sin embargo, cuando se queman materiales lignocelulósicos, combustibles complejos o materiales carbonados con una cantidad importante de átomos de distinta naturaleza, el proceso de separación se convierte en una piedra angular. Como un ejemplo de lo anterior, uno de los solventes empleados en una absorción física es el Selexol (dimetil éter de polietilenglicol) que tiene gran eficiencia $(98 \%$ en masa de recuperación), pero la presencia de hidrocarburos o ácido sulfhídrico interviene en la absorción [22].

\section{Conclusiones}

Los esquemas de separación de $\mathrm{CO}_{2}$ en procesos de combustión están íntimamente ligados a las condiciones de combustión, y con base en ellos es que deben diseñarse. Existen tres métodos de combustión con captura de $\mathrm{CO}_{2}, \mathrm{y}$ están basados en el momento cuando se hace la captura o del material oxidante. El análisis realizado para la combustión de metanol corresponde a simulaciones preliminares 
donde se utiliza un enfoque de poscombustión y el estudio de la separación por ruta física y química. Los resultados indican que para combustiones completas del metanol la mejor ruta está determinada por la eficiencia en la recuperación. Es necesario estudiar otras rutas y otros combustibles con el fin de determinar si la separación física o química es la más apropiada para este tipo de corrientes.

\section{REFERENCIAS}

[1] J. F. Isaza, Cambio climático, glaciaciones y calentamiento global. Bogotá: Fundación Universidad de Bogotá Jorge Tadeo Lozano, 2007.

[2] C. P. Tsokos and Y. Xu, "Modeling carbon dioxide emissions with a system of differential equations", Nonlinear Analysis: Theory, Methods \& Applications, vol. 71, pp. e1182-e1197, 2009.

[3] J. Otero, "Procesos de captura de CO2", Ambienta, vol. 51, pp. 40-47, 2006.

[4] J. C. M. Pires et al., "Recent developments on carbon capture and storage: An overview", Chemical Engineering Research and Design, vol. In Press, Corrected Proof.

[5] H. Yang et al., "Progress in carbon dioxide separation and capture: A review", Journal of Environmental Sciences, vol. 20, pp. 14-27, 2008.

[6] I. Omae, "Aspects of carbon dioxide utilization", Catalysis Today, vol. 115, pp.33$52,2006$.

[7] P. Ruiz, "Aplicación del dióxido de carbono supercrítico al procesado de alimentos: Nata, subproductos del refinado de aceites vegetales y zumo", Doctorado en Farmacia, Nutrición y Bromatología II, Universidad Complutense de Madrid, Madrid, 1996.

[8] B. Bassegoda, Técnicas de contrucción III. Barcelona: Editores Técnicos y Asociados S.A, 1982.
[9] V.Bermúdez, Tecnología energética. Valencia: Universidad Politécnica de Valencia, 2000.

[10] K. Damen et al., "A comparison of electricity and hydrogen production systems with $\mathrm{CO} 2$ capture and storage. Part A: Review and selection of promising conversion and capture technologies", Progress in Energy and Combustion Science, vol. 32, pp. 215246, 2006.

[11] H. L. MacLean and L. B. Lave, "Evaluating automobile fuel/propulsion system technologies", Progress in Energy and Combustion Science, vol. 29, pp. 1-69, 2003.

[12] R. A. Dobbins et al., "Polycyclic aromatic hydrocarbons in flames, in diesel fuels, and in diesel emissions", Combustion and Flame, vol. 144, pp. 773-781, 2006.

[13] M. Xu et al., "Status of trace element emission in a coal combustion process: a review", Fuel Processing Technology, vol. 85, pp. 215-237, 2004.

[14] I. Pietro, Captura y almacenamiento de $\mathrm{CO}_{2}$ procedente de instalaciones de combustión para la generación de energía eléctrica. Oviedo: Universidad de Oviedo, 2009.

[15] C. A. Scholes et al., "CO2 capture from pre-combustion processes--Strategies for membrane gas separation", International Journal of Greenhouse Gas Control, vol. 4, pp. 739-755, 2010.

[16] M. R. M. Abu-Zahra et al., "New process concepts for $\mathrm{CO} 2$ post-combustion capture process integrated with co-production of hydrogen", International Journal of Hydrogen Energy, vol. 34, pp. 3992-4004, 2009.

[17] A. Padurean et al., "Multicriterial analysis of post-combustion carbon dioxide capture using alkanolamines", International Journal of Greenhouse Gas Control, vol. In Press, Corrected Proof.

[18] F. López and A. Sánchez, "Análisis termoeconómico de procesos de captura de 
$\mathrm{CO}_{2}$ en ciclos combinados", presented at the IV Conferencia Científica Internacional de Ingeniería Mecánica, Santa Clara, 2006.

[19] S. Sabugal, "Captura y almacenamiento de $\mathrm{CO}_{2}$ a partir de oxicombustión" presentado en la Cátedra Rafael Mariño, Madrid, 2006.

[20] Methanol Institute, Mezclas de gasolina $y$ metanol: Combustible alternativo para los automóviles actuales y octanaje de combustión más limpia para las refinerías de petróleos actuales, Methanol Institute, Bruselas, 2009.
[21] T. Kausel, "El metanol: Un combustible sintético con alto potencial para la nueva Región de los Ríos y el país", en: Demandas y desafios del sector Energético en la Región de los Ríos, Valdivia, 2007.

[22] S. Nourouzi-Lavasani et al., "Energy and Hydrogen Coproduction from (Athabasca Bitumen) Coke Gasification with $\mathrm{CO} 2$ Capture", Industrial \& Engineering Chemical Research, vol. 47, pp. 7118-7129, 2008. 